\section{Do young chimpanzees have extraordinary working memory?}

\author{
Peter Cook and Margaret Willon \\ University of California, Santa Cruz, California
}

Do chimpanzees have better spatial working memory than humans? In a previous report, a juvenile chimpanzee outperformed 3 university students on memory for briefly displayed digits in a spatial array (Inoue \& Matsuzawa, 2007). The authors described these abilities as extraordinary and likened the chimpanzee's performance to eidetic memory. However, the chimpanzee received extensive practice on a non-time-pressured version of the task; the human subjects received none. Here we report that, after adequate practice, 2 university students substantially outperformed the chimpanzee. There is no evidence for a superior or qualitatively different spatial memory system in chimpanzees.

Do chimpanzees have better spatial working memory than humans? In a highly publicized study, a juvenile chimpanzee, Ayumu, performed substantially better than university students on memory for digits displayed for $210 \mathrm{msec}$ in a spatial array (Inoue \& Matsuzawa, 2007). The authors further observed that Ayumu could pause several seconds before responding - for example, in reaction to environmental sounds - and still maintain high performance. The authors described these abilities as "extraordinary" and likened them to eidetic (so-called "photographic") memory. The findings were reported by The New York Times, the BBC, NPR, and all four U.S. television networks, as well as in the pages of Science.

However, Ayumu was the only 1 of 6 chimpanzees to achieve this level of performance. Furthermore, Inoue and Matusawa gave their 6 chimpanzees extensive training on a non-time-pressured version of the task, wherein the digits did not disappear until the subject touched the first digit. Training involved approximately 200 trials per day, 5 or 6 days a week, and began in 2005. At the time the article was published in 2007, 5 of the 6 chimpanzees (those other than Ayumu) were still being trained. From this, we can infer that training was quite extensive. In contrast, the human subjects received almost no practice of any sort.

In a subsequent study, Silberberg and Kearns (2009) showed that humans can match Ayumu's performance when they get extensive time-pressured practice - that is, practice at the extremely short 210 -msec stimulus duration that was used for the final test. This finding, while casting some doubt on the conclusions of Inoue and Matsuzawa, does not decisively overturn them. It is perhaps unsurprising that human performance improved for the 210-msec task after practice on the exact same task. In contrast, Ayumu's success occurred with no prior practice at $210 \mathrm{msec}$. Instead, Ayumu's practice sessions allowed the animal to observe the display at leisure until initiating the response. Inoue and Matsuzawa reported that the average latency before responding was $650 \mathrm{msec}$ - far greater than the exposure time used by Silberberg and Kearns for practice. Thus, parallel performance in humans and chimpanzees under comparable conditions has not yet been demonstrated.

In the present article, we report that, with practice similar to that undertaken by Ayumu, our human subjects not only matched but outperformed Ayumu. Furthermore, human subjects can maintain high performance with short delays, demonstrating an ability similar to that reported for Ayumu. Finally, a pattern mask disrupted performance, suggesting that facility in this task is supported by ordinary processes of iconic memory storage or retinal afterimage. In sum, there is no evidence for a superior or qualitatively different spatial memory system in chimpanzees.

\section{METHOD}

Two undergraduate research assistants, T.E. and J.S., served as subjects as part of their lab responsibilities. Following Inoue and Matsuzawa, stimuli were five digits chosen randomly for each trial, without replacement, from the digits 1-9. These were displayed simultaneously in random locations in an invisible $8 \times 5$ grid. After a fixed stimulus duration (see below), the digits were replaced by white rectangles. The task was to click the rectangles in the numerical order of the digits they replaced. Each correct click caused the rectangle to disappear, and an incorrect click ended the trial. Performance was measured in terms of the number of trials finished completely correct.

\section{Pretest}

In a procedure as nearly identical as possible to that of Inoue and Matsuzawa's shortest duration condition, each subject ran two blocks of 50 trials at a $210-\mathrm{msec}$ stimulus duration. The first block was discarded as practice. Performance on the second block was $46 \%$ and $38 \%$ percent for subjects J.S. and T.E., respectively, which is comparable to the human performance of approximately $40 \%$ reported by Inoue and Matsuzawa.

\section{Training}

As noted above, the chimpanzee exposure times during training were approximately $650 \mathrm{msec}$. In contrast, humans tested by Inoue and Matsuzawa on the training procedure chose to look at the display much longer (sometimes over $7 \mathrm{sec}$ ), but were more accurate than the chimpanzees, indicating a speed-accuracy trade-off. Clearly, humans, left to their own devices, would give themselves a training experience different from that chosen by Ayumu.

Therefore, in order to provide training at durations comparable to those experienced by Ayumu, we trained our subjects at a stimulus duration of $650 \mathrm{msec}$. All of the training used a display of five digits. Subjects ran 4 blocks of 50 trials per day, 5 days a week, until a total of 300 blocks was reached. Performance on the last 4 blocks of training was $100 \%$ for T.E. and $98 \%$ for J.S. 


\section{Posttest}

Following training, subjects were run on one practice block and four test blocks at the 210 -msec stimulus duration, just as in the pretest.

In addition, subjects were tested in a delay condition, in an attempt to duplicate Ayumu's ability to delay responding. Since Ayumu showed this ability during the training phase, the delay condition was run with the 650 -msec stimulus duration. After the white rectangles appeared, there was a delay of $5 \mathrm{sec}$, during which the computer would not accept responses. A tone at the end of the $5 \mathrm{sec}$ signaled that responding could begin. Again, there was one practice block and four test blocks.

Inoue and Matsuzawa used a checkerboard pattern mask in some of their chimpanzee training sessions in order to "completely suppress the possible afterimage" (Inoue \& Matsuzawa, 2007, online supplemental methods), but they did not report performance levels. However, a checkerboard is not an appropriate pattern mask for digits (Breitmeyer \& Öğmen, 2007). Therefore, we ran one additional test condition at the $210-\mathrm{msec}$ stimulus duration, in which a complex pattern mask made up of straight and curved white lines resembling the elements of digits was used in place of the plain white rectangle. Such a mask should disrupt performance that relies on iconic memory or retinal afterimages. As in the two previous test conditions, one practice block was followed by four test blocks.

\section{RESULTS}

Performance on the posttest was $94 \%$ for T.E. and $96 \%$ for J.S., substantially better than Inoue and Matsuzawa's human subjects and better than Ayumu (see Figure 1). A chi-square test showed that the pooled data of T.E. and J.S. differed significantly from Ayumu's $\left(\chi^{2}=47.6, p<\right.$ $.001)$. Performance in the delay condition was $93 \%$ for

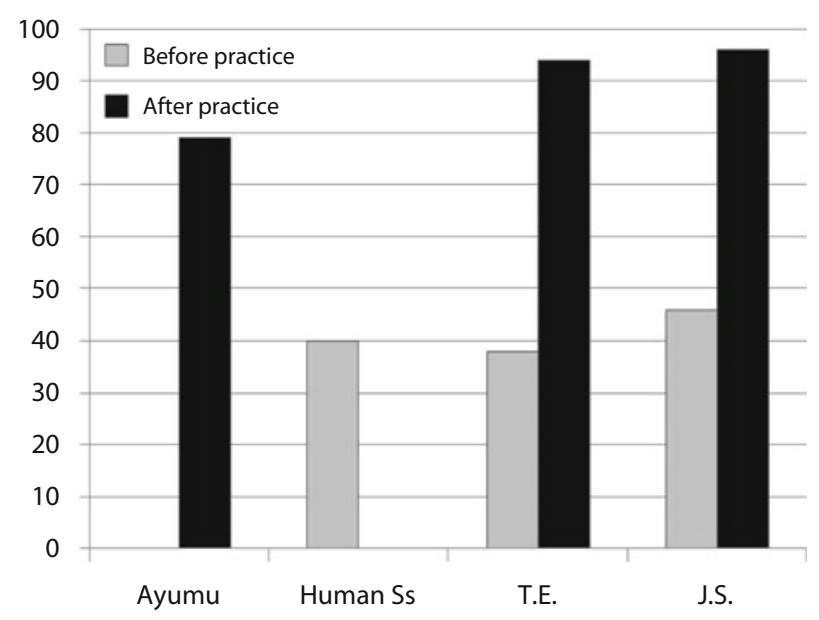

Figure 1. Percentages of trials completely correct on the 210msec stimulus duration posttest, with and without practice. Ayumu's and the human subjects' results are from Inoue and Matsuzawa (2007). Values for T.E. and J.S. are from the present study.
T.E. and $96.5 \%$ for J.S., which does not differ from performance in the no-delay posttest. Performance in the pattern-mask condition was $77 \%$ for T.E. and $81.5 \%$ for J.S., significantly lower than in the basic posttest $\left(\chi^{2}=\right.$ 44.2, $p<.001)$.

\section{DISCUSSION}

Our results show that practice with a 650 -msec stimulus duration is sufficient to improve task performance at $210 \mathrm{msec}$ without notable prior exposure at the shorter duration and that humans are capable of better performance on this task than even the most talented chimpanzee. In addition, humans can tolerate a delay on this task with no impact on performance, undercutting any claim that Ayumu's ability is extraordinary in this regard. Furthermore, results with a pattern mask indicate that performance on this task relies substantially on ordinary processes of iconic memory or retinal afterimages. In sum, there are no grounds to believe that chimpanzees have an extraordinary working memory ability reminiscent of eidetic imagery. Incidentally, the existence of eidetic imagery is itself controversial, and the concept has been persistently "defined downward" since the failure to validate early claims of photographic memory (Gray \& Gummerman, 1975).

Inoue and Matsuzawa's focus on Ayumu's apparently superior performance obscures the genuine interest of their data and what they can tell us about primate cognitive processing. Points of interest include better performance by juveniles than by adults, good performance without the aid of verbal rehearsal, and the ability to resist distraction, suggesting rapid consolidation. We hope that future research will focus on these more nuanced questions.

\section{AUTHOR NOTE}

Correspondence concerning this article should be addressed to P. Cook, Department of Cognitive Psychology, University of California, Santa Cruz, CA 95064 (e-mail: pcook@ucsc.edu).

\section{REFERENCES}

BreitMeyer, B. G., \& ÖĞMEN, H. (2007). Visual masking. Scholarpedia, 2(7), 3330. doi:10.4249/scholarpedia.3330

Gray, C. R., \& Gummerman, K. (1975). The enigmatic eidetic image: A critical examination of methods, data, and theories. Psychological Bulletin, 82, 383-407.

Inoue, S., \& MatsuzaWa, T. (2007). Working memory of numerals in chimpanzees. Current Biology, 17, R1004-R1005.

Silberberg, A., \& Kearns, D. (2009). Memory for the order of briefly presented numerals in humans as a function of practice. Animal Cognition, 12, 405-407.

(Manuscript received October 28, 2009; revision accepted for publication March 9, 2010.) 